\title{
Managing depression in primary care
}

\author{
Public confidence needs to be restored after concerns over the safety of SSRIs
}

$\mathrm{D}$ epression is a condition of "particular concern, which costs lives and affects the quality of life," according to UK prime minister Tony Blair. ${ }^{1}$ Nine out of 10 depressed patients are treated only in primary care, ${ }^{23}$ and up to two thirds of suicide victims contact a general practitioner in the four weeks before the death. ${ }^{4}$ For years, however, general practitioners have been criticised for failing to deal adequately with depression. Like hypertension, depression is subject to a rule of halves-only half of depressed patients seek help from doctors, half are detected in primary care, half receive treatment with only half completing it: fewer than $10 \%$ finish a therapeutic course of treatment. $^{5}$ A range of initiatives, often erroneously based on educational models for general practitioners, has aimed to improve detection rates and to increase the appropriateness of prescribing in depression with variable success, including a spectacular failure, in Hampshire, to influence general practitioners' behaviour at all. ${ }^{6}$

There are many reasons why depression goes unrecognised in primary care. ${ }^{7}$ Patients may attribute symptoms wrongly, may present instead with physical symptoms, may not realise that they need treatment, ${ }^{7}$ and may attend their doctors' practices too infrequently. ${ }^{8}$ General practitioners may have negative attitudes to mental health problems and not feel responsible for dealing with them, may lack time, may lack facilities in poorly organised practices, may have inadequate consulting skills, and may be deterred by the workload of long term treatment and monitoring. Before selective serotonin reuptake inhibitors (SSRIs) became available, general practitioners were legitimately concerned about the side effects of treatment, particularly with the older tricyclic antidepressants. Although more caution in prescribing has recently been advocated, ${ }^{\text {w1 }}{ }^{\text {w2 }}$ SSRIs initially offered a new dawn for depression treatment in general practice, with drugs that were relatively free of side effects.

The UK Committee on Safety of Medicines, informed by a report from an expert working group, issued guidance on the safety of SSRIs in December 2004. ${ }^{\text {w3 }}$ The committee's advice, which has been incorporated into information for prescribers and patients, concerns withdrawal reactions, dose changes, and suicidal behaviour and recommends that treatment with venlafaxine, a serotonin and noradrenaline reuptake inhibitor, should be initiated only by specialist mental health practitioners, including general practitioners with a special interest in mental health.

The response to these warnings has been extraordinary. The media have caricatured general practitioners as prescribing from the hip, using SSRIs in depression like antibiotics in sore throat. Worse, general practitioners have been portrayed as incapable of judging how to prescribe appropriately. At least one professor of psychiatry has suggested, not for the first time, that all depressed patients requiring drug treatment should be under the care of a psychiatrist.
This suggestion would bring the hospital system to its knees within days.

Despite this overreaction, national policy continues to support the strategy that depression should be managed in primary care. On the same day the Committee on Safety of Medicines released its report, the National Institute for Clinical Excellence (NICE) published evidence based guidelines supporting a careful, stepped approach to managing depression in primary care. ${ }^{\mathrm{w} 4}$ Depending on the severity of depression, the guidelines recommend watchful waiting, exercise, self help, and psychological therapies, as well as antidepressant treatment. First line treatment for moderate to severe depression, according to NICE, should be with SSRIs, although these are probably of little use in mild depression. The stepped approach will involve the whole primary care team, including the 1000 new graduate mental health workers announced in the NHS Plan for England. A pilot scheme in the Trent region of England has been described. ${ }^{\text {w5 }}$

The general public, surveyed a decade ago, preferred to see general practitioners rather than psychiatrists for depression and to receive psychological treatments and counselling rather than drug treatment. ${ }^{9}$ Many general practices still lack such resources, but could do much more with the help of nurses and graduate health workers. Practice nurses have recently improved access to treatment and increased patients' satisfaction by running an open access depression service in south London. ${ }^{10}$ Nurses and graduate mental health workers could encourage depressed patients to seek help in primary care and provide advice on nutrition, alcohol, sleep hygiene, and exercise, teach patients to use self help materials, and deliver brief psychological interventions, and to direct patients to voluntary groups such as Depression Alliance. Computerised psychological treatments may solve the problem of low availability of services for some. ${ }^{11}$

Given that NHS strategy sees primary care as the right place for most depression services, the absence of depression from the list of chronic diseases that general practitioners are specifically paid to manage through the new General Medical Services contract is a glaring omission. ${ }^{\text {w6 }}$ However, the Primary Care Programme of the National Institute of Mental Health in England is committed to helping practices to improve depression care, by providing guidance on locally enhanced services for depression and to improving existing training for general practitioners with a special interest in mental health, ${ }^{12}$ although few of these enhanced services have been established so far.

Public confidence in the ability of general practitioners to manage depression has been knocked by current concerns about SSRIs, exaggerated in the media. Better support for self help, wider provision of effective psychological services, and more appropriate

Additional references w1-w6 are on bmj.com 
use of antidepressants in primary care, and more urgent attention by NHS planners, should help to restore that confidence.

Andre Tylee professor of primary care mental health Health Services Research Department, Institute of Psychiatry, London SE5 8AF

\section{Roger Jones Wolfson professor of general practice}

Department of General Practice and Primary Care, GKT School of Medicine, London SE11 6SP

(roger.jones@kcl.ac.uk)

Competing interests: None declared.

1 Blair T. Message from the prime minister. In: Dawson A, Tylee A, eds Depression: social and economic timebomb. BMJ Publishing Group, London, 2001:ix.

2 Shepherd M, Cooper B, Brown AC, Kalton G. Psychiatric illness in general practice. London: Oxford University Press, 1966.

3 Goldberg D, Huxley P. Common mental disorders. A biosocial model. London: Routledge, 1992

4 Pirkis J, Burgess P. Suicide and recency of health care contacts. $\mathrm{Br} J$ Psychiatry 1998;173:462-74.
5 Lépine JP, Gastpar M, Mendlewicz J, Tylee A. Depression in the community: the first pan-European study DEPRES (Depression Research in nity: the first pan-European study DEPRES (Depression
Europe Society). Int Clin Psychopharmacol 1997;12:19-29.

6 Thompson C, Kinmonth AL, Stevens L, Peveler RC, Stevens A, Ostler KJ, et al. Effects of clinical-practice guideline and pradtice-based education on detection and outcome of depression in primary care: Hampshire Depression project randomised controlled trial. Lancet 2000;355:185-91.

7 Walters P, Tylee A. In: Maj M, Lopez-Ibor J, Sartorius N, Sato M, Okasha A, eds. Early detection and management of mental disorders. World Psychiatric Association. Chichester: John Wiley, 2005.

8 Bushnell J on behalf of the MaGPIe Research Group. Frequency of consultations and general practitioner recognition of psychological symptoms. Br J Gen Pract 2004;54:838-42.

9 Priest RG, Vize C, Roberts A, Tylee A. Lay people's attitudes to treatment of depression: results of opinion poll for Defeat Depression Campaign of depression: results of opinion poll for Deft

10 Symons L, Tylee A, Mann A, Jones R, Plummer S, Walker M, et al. Improving access to depression care: descriptive report of a multi-disciplinary primary care pilot service. Br J Gen Pract 2004;54: 679-83.

11 Proudfoot J, Ryden C, Everitt B, Shapiro D, Goldberg D, Mann A, et al. Clinical effectiveness of computerised cognitive behavioural therapy for anxiety and depression primary care. A randomized controlled trial. $\mathrm{Br} J$ Psychiatry 2004; 185:46-54.

12 Enhanced Services Specification for depression under the new GP contract: a commissioning guidebook. National Institute for Mental Health in England. $\mathrm{http} / / / \mathrm{kc}$.nimhe.org.uk/index.cfm?fuseaction = Group.viewPost\&intPost $\mathrm{ID}=20733$ \&intGroupID = 209 (accessed 22 Mar 2005).

\section{Aortic stenosis}

\section{Is common, but often unrecognised}

$\mathrm{A}$ ortic valve disease is common. In Western populations, about $25 \%$ of people aged over 65 have aortic sclerosis, and 3\% over 75 have severe stenosis. ${ }^{1}$ In 2003, 6028 aortic valve operations were done in Britain and Ireland compared with 25277 for isolated coronary disease. ${ }^{2}$ Despite this the diagnosis may often be missed. Probably half are still diagnosed at postmortem examination, and $5 \%$ of operations are performed at end stage. ${ }^{3}$ Unrecognised aortic stenosis is an important cause of anaesthetic and obstetric mortality.

The overwhelming cause of aortic valve disease in Europe is calcific degenerative disease, and about 2\% of the population have congenitally bicuspid aortic valves. Rheumatic disease is now rare. Stenosis is differentiated from sclerosis, when a restriction of cusp movement and a raised transaortic peak velocity are seen on echocardiography. Around $16 \%$ of patients with sclerosis progress to stenosis in seven years. ${ }^{4}$ The early lesions of calcific degenerative disease resemble atheroma of the coronary artery. Many of the risk factors for aortic stenosis are common to other atherosclerotic processes, and one would expect treatment with statins to reduce the rate of progression. However, a recent, small, randomised study of atorvastatin was negative. ${ }^{5}$ Aortic valve disease is also a marker for coronary disease and coronary events. The incidence of myocardial infarction is $6.0 \%$ over five years in septuagenarians with normal aortic valves, $8.6 \%$ with aortic sclerosis, and $11.3 \%$ with aortic stenosis. ${ }^{7}$ Current randomised trials, including the simvastatin and ezetimibe in aortic stenosis (SEAS) study will test the effect of lipid lowering on both cardiovascular events and the progression of aortic stenosis.

One reason for failing to make a diagnosis is that the clinical signs can be difficult to interpret. Although recommended indications for echocardiography grade of the murmur may not always reflect the severity of the disease. ${ }^{9}$ All typical textbook guides are of limited value, particularly when the examiner is inexperienced or does not have specialist training in cardiology. ${ }^{9}$ A normal or high blood pressure with a normal pulse pressure is often used to dismiss the possibility of severe aortic stenosis. However, only $7 \%$ of patients referred for surgery have a pulse pressure of less than $35 \mathrm{~mm} \mathrm{Hg}$, and the second sound and carotid upstroke may also be normal. ${ }^{9}$ The signs of aortic stenosis were originally described in young patients when the dominant cause was rheumatic disease. Calcific degenerative disease in older people is often associated with systemic hypertension, coronary disease, and arteriosclerosis, all of which can confound the effects of aortic stenosis on the circulation and therefore modify the clinical signs.

Another reason for failing to recognise aortic stenosis is that most patients have a long asymptomatic period and may therefore not seek medical attention. During this period, the mortality is low. Guidelines recommend surgery when symptoms develop since mortality then rises sharply. The median survival is 4.5 years with exertional chest pain, 2.6 years with exertional dizziness, and one year with overt heart failure. ${ }^{10}$ Around $10-15 \%$ of patients die soon after the onset of symptoms, giving little time to make the diagnosis and organise surgery.

The aim should therefore be to make the diagnosis while the patient is still apparently asymptomatic. For those who are truly asymptomatic, echocardiography is increasingly being used to predict the likely onset of symptoms so as to plan elective surgery. The use of blood testing for brain natriuretic peptide is also being explored. Most patients should also have a treadmill exercise test because unexpected symptoms will show up in up to half of them. ${ }^{11}$ Symptoms may not be apparent in daily life because the patient may be sedentary or 